\title{
Design and optimization of the concept of a passenger train storage system
}

Lucian Cucu ${ }^{1}$, Marilena Stoica ${ }^{2}$, Ionel Simion ${ }^{3}$, and Gina Florica Stoica $^{4}$

1,2,3,4 University Politehnica of Bucharest, Machine Elements Department, Bucharest, Romania

\begin{abstract}
The aim of this paper is to design a passenger train storage system. It concerns with the idea of safe storage in public transportation. The design consists of developing a new type of storage system complying with the standards of the European Union (EU) that can improve the railroad transportation system.
\end{abstract}

\section{Problem statement}

The requirements of passenger train storage system involves a light structure, easy manufacturing, using standardized components, easy access, user friendly [1]. The actual design of the train car does not offer any capabilities of designing the internal volume [2]. The solution started with the parametrization of the internal volume of the train car by redesigning the internal space in order to accept a new modular design storage without interfering with the train dynamics. The first iteration of the study was applying the revolver mechanism study with an electromagnetically mechanism of the storage system. For the optimization, we have studied the possibility to use a chain mechanism for a slow gearing in order to minimize the inertia moments. The solution adopted was an external attachment to the train car using the revolver concept developed in order to achieve a good reliability of the passenger train system. The model involves designing the exterior structure (using the standard dimension of the train car), the exoskeleton, and the storage units. The study was made in designing the internal volume of the storage system in order to accept the largest luggage existent on the market.

\section{Theoretical background}

Starting with the existing dimensions of the train car (figure 1), which are standardized and cannot be modified extensively, the study proposes a new design of the storage space, in order to comply with the EU (European Union) rail road design standards [3]. The study begun with elements related to morphology, functionality and user interaction.

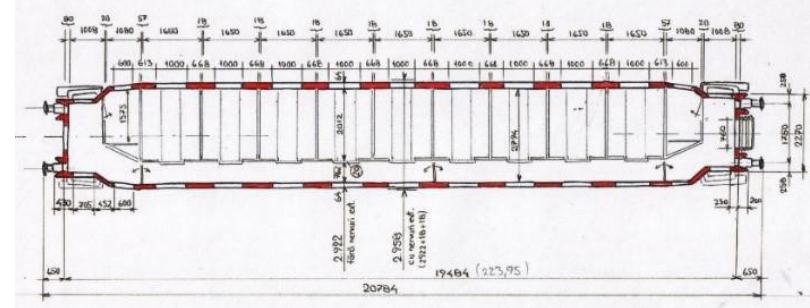

Fig.1 Standard passenger train car dimensions and layout

The proposal is shown in figure 2, which describes a fixed modular structure that can be attached to the both end of the train car, making single unit with the train car.

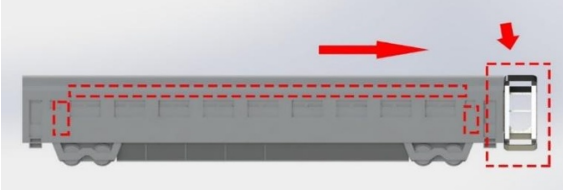

a) side view

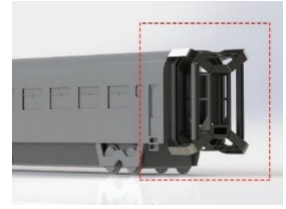

b) Posioning detail

Fig. 2 Reconfiguring the storage space on the exterior of the train car 
This study has two main aspects regarding the optimization of public transportation. The first one refers to a system storage that offers modularity through which it can be attached to other different passenger train cars. The second aspect considers three directions:

- the parametrization of the internal storage space for different types of luggages (dimensions and designs);

- optimization of the whole system in order to be adapted to other public transportation variants;

- can be used like a storage system in public spaces.

\section{Description of the internal volume of passenger train storage system}

The normal internal storage space of a train car is limited to small to medium luggages with narrow passageways, which can be difficult for the user to travel with large and heavy luggages. The new system should offer acces on both sides in order for the user to access their luggages on different train stations. There are systems, which offer this functionality like the Revolver system and Carousel parking system.

The iteration for the final concept is shown in figure 3 .
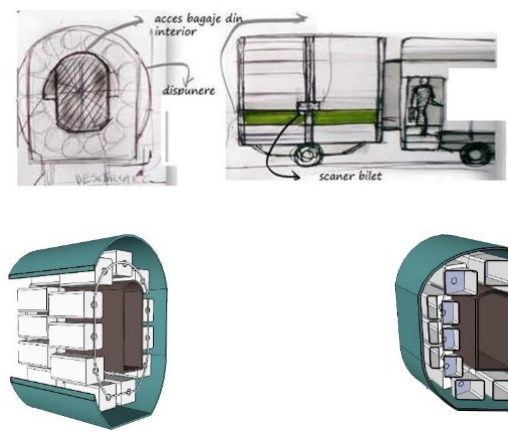

a) Concept

sketch of the

module
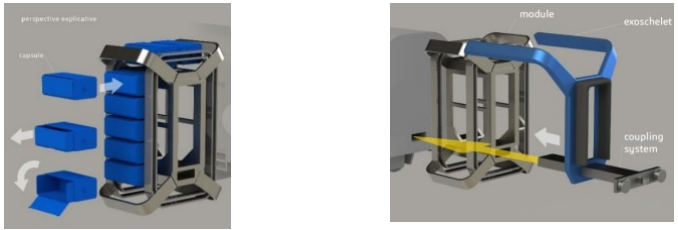

c) Final

concept

Fig. 3 Concept iterations

The internal structure it is composed with metallic struts in which it's placed an subassembly of 14 capsules mounted in a transportation system capable to take and deliver the luggages at different height levels according to the architecture of the train station.

The structure morphology allows a passage space in order for the passenger to go from car to car. The structure is assembled to the train car using the standard coupling system. The 14 capsules subassembly is moved by an electric system capable to move the luggages on a pre-established path.

The closing and opening system has 3 elements and offers access on both sides for users according to train station architecture. The frame that it is attached to the transportation system on the module. The drawer that can move half way on both sides. The door that in the open position forms an access ramp for a better accessibility. The design of the transportation system started with the constructive predimensioning of the module taking in consideration the maximum loads applied to it. A chain transmission was chosen on which the capsule where attached. The efforts from the storage module are transmitted using a conveyor-based system. The conveyors usually are linear, horizontal, helicoidally and vertical. The system requires a closed loop, which has translational and rotational movement. The capsule trajectory needs to be accurate and it needs to keep the luggage at a level plain. In order to achieve that, a stabilizer solution is needed. 


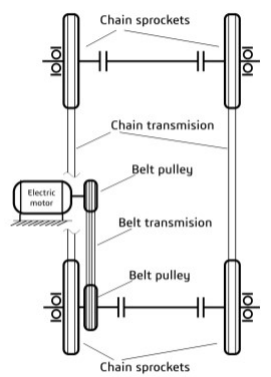

\author{
a) Concept sketch \\ of the system \\ transmission
}
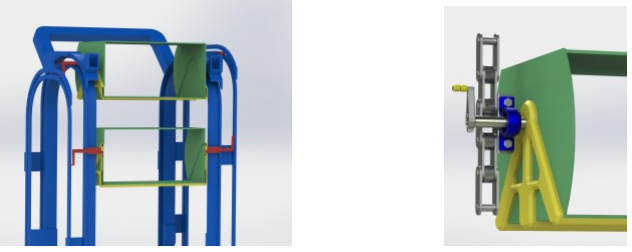

b) Stabilizer

solution
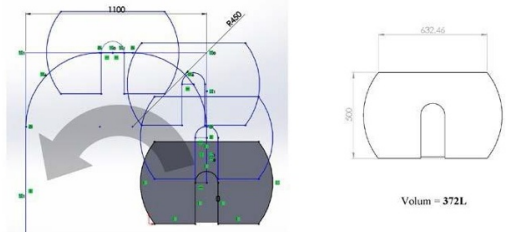

c) close loop

trajectory of the

capsule

Fig. 4 Design of the transmission and stabilizer

For a full load on the module each capsule fully loaded, the maximum load on the chain is $43 \mathrm{kN}$. With this value the NR $\mathrm{C} 2162 \mathrm{H}$ chain was chosen.

\title{
4. Conclusions
}

The study was made using Solidworks and Fusion 360. The optimization was made considering 3 main factors: the loads, materials and the type of assembly. For further studies, an important factor, it is also the user safety in the day-by-day usage scenario of the storage system regarding the current world threats. In order to perform this study we will make an ergonomic study to achieve a good user accessibility.

\section{Bibliography}

[1] P. E, "Neufert, Architects' Data", vol. 3, pp. 426-432.

[2] R. V, M. J. F and P. R, "Rail and multi-modal transport," Research in transportation economics, vol. 41, 2013.

[3] N. A. Kruger, "Estimating traffic demand risk-A multiscale analysis" Swedish National Road and Transportation Research Institute, 2012 